\title{
Reliability of biceps femoris and semitendinosus muscle architecture measurements obtained with ultrasonography
}

\author{
Viviane Bastos de Oliveira, Simone Peres Carneiro, Liliam Fernandes de Oliveira*
}

\begin{abstract}
Introduction: Currently, little attention is given to the muscle architecture reliability studies of the hamstring using a robust statistical. Our purpose was to determine the reliability of ultrasound measurements of muscle thickness, fascicle length and pennation angle of the biceps femoris and semitendinosus muscles, including heteroskedasticity and internal consistency analyses. Methods: Two images of biceps femoris and semitendinosus at $50 \%$ of the thigh length were acquired from 21 volunteers, in two visits. The parameters were measured three times in each image, and for each muscle. The reliability was analyzed by the intraclass correlation coefficient (ICC) and Cronbach's alpha ( $\alpha$ Cronbach). The relative standard error of the measurements $(\% \mathrm{SEM})$ were calculated and Bland-Altman plots were generated. Results: All parameters presented excellent ICC for the three repeated measurements (ICC from 0.93 - 0.99) and moderate to excellent reliability intraday (ICC from $0.70-0.95)$ for both muscles. The present study indicates that ultrasound is a reliable tool to estimate the biceps femoris fascicle length $(\mathrm{ICC}=0.97, \alpha \mathrm{Cronbach}=0.98, \% \mathrm{SEM}=7.86)$ and semitendinosus $(\mathrm{ICC}=0.90$, $\alpha \mathrm{Cronbach}=0.95, \% \mathrm{SEM}=7.55)$, as well as the biceps femoris muscle thickness $(\mathrm{ICC}=0.89, \alpha \mathrm{Cronbach}=0.94$, $\% \mathrm{SEM}=10.23)$ and semitendinosus muscle thickness $(\mathrm{ICC}=0.87, \alpha \mathrm{Cronbach}=0.93, \% \mathrm{SEM}=1.35)$. At last, biceps femoris pennation angle $(\mathrm{ICC}=0.93, \alpha \mathrm{Cronbach}=0.96$ and $\% \mathrm{SEM}=4.36)$ and semitendinosus $(\mathrm{ICC}=0.96, \alpha \mathrm{Cronbach}=0.98$ and $\% \mathrm{SEM}=4.25)$ also had good repeatability. Conclusion: Ultrasonography show good repeatability in estimating of muscle architecture parameters.
\end{abstract}

Keywords: Reliability, Hamstring, Ultrasound, Muscle architecture.

\section{Introduction}

Muscle architecture is defined as the arrangement of muscle fibers and plays an important role in muscle biomechanics studies (Lieber, 2010). Fiber length, pennation angle and muscle thickness are commonly measured as architecture parameters (Blazevich et al., 2006; Gomes et al., 2010; Lima et al., 2012; Martins et al., 2012). Technological advances in imaging techniques, as magnetic resonance imaging and ultrasound, enable to estimate muscle architecture parameters in vivo (Blazevich et al., 2006; Chleboun et al., 2001; Lima et al., 2012). The architecture parameters can be well visualized by ultrasound at rest or during contraction (Gomes et al., 2010; Martins et al., 2012; Potier et al., 2009; Timmins et al., 2015). The reliability of muscle architecture measurements assessed using ultrasound is reported in many studies with different methodologies involving repeated measures between images, sessions, and raters (Legerlotz et al., 2010; Lima et al., 2012). These studies have been important to determine a reproducibility of muscle architecture parameters which are measured very often in training and rehabilitation protocols. Concerning the lower limbs, reliability studies in vivo are described for the knee extensors (Lima et al., 2012; Noorkoiv et al.,
2010) and gastrocnemius muscles (Legerlotz et al., 2010; Martins et al., 2012). The reliability of muscle architecture measurements of the knee flexors, using ultrasound, is less examined in vivo (Chleboun et al., 2001; Gomes et al., 2010; Timmins et al., 2015).

Reliability studies of knee flexors focused mainly on the biceps femoris long head muscle, and estimative of the intraclass correlation coefficient (ICC) to fascicle length, muscle thickness and pennation angle ranged around $0.87-0.95$ (Chleboun et al., 2001; Timmins et al., 2015). For these parameters, it was found coefficient of variation (CV) below $5 \%$ at rest. Kellis et al. (2009) compared the muscle thickness, fascicle length and pennation length measurements of the semitendinosus and the biceps femoris long head muscles using a caliper to validate ultrasound images of a cadaveric limb. The authors showed good reliability for all parameters (ICC $>0.79$ ) and standard error of measurement (SEM) between 4.7 to $9.7 \%$. Measurements of whole muscle thickness of the knee flexors reported by Gomes et al. (2010) was moderate (ICC $=0.55$ ), with $6.66 \%$ of CV and $9.6 \%$ of SEM.

Statistical estimators as SEM and ICC are commonly referred in reliability studies. As can be 
seen in the previous paragraph, statistical estimators as SEM presuppose absence of data heteroskedasticity, which refers to the proportional increase of the data magnitude with the random errors (Atkinson and Nevill, 1998). Furthermore, the internal consistency of the measurements estimates how items are correlated within group data, but has often been neglected in those studies (Hopkins, 2000). Based on this, the aim of this study is to analyze the absolute test-retest reliability of the muscle thickness, fascicle length and pennation angle of the biceps femoris long head and semitendinosus muscles using ultrasound. The absence of heteroskedasticity and internal consistency were included in the statistical analysis.

\section{Methods}

\section{Participants}

Twenty-one $(\mathrm{N}=21)$ healthy volunteers participated in this study. The volunteers were 6 women and 15 men aged $28.08 \pm 4.61$ years, body mass of $74.03 \pm 13.49 \mathrm{~kg}$, and height of $1.74 \pm 0.08 \mathrm{~m}$. They did not have any diseases in the lower limbs. The study was approved by the Ethics and Research Committee of the Clementino Fraga Filho University Hospital ( $n^{\circ}$ 023/11). Informed consent was obtained from all individual participants included in the study. Volunteers were instructed not to perform any type of vigorous physical activity involving the lower limbs during the test.

\section{Ultrasound measurements}

Images acquisition were performed by the same trained examiner with an ultrasonographic system operating in B-Mode (EUB-405; Hitachi, Tokyo, Japan) with a $7.5 \mathrm{MHz}$ linear array probe. With the participants at standing position, the examiner marked one point at $50 \%$ of the length of the thigh, determined by the distance between the greater trochanter and head of the fibula. After that, the subject laid with the legs relaxed in prone position for 15 minutes before image acquisition on the right leg.

The protocol was repeated in two days (interdays reliability), with a 48 -hour interval between visits. Two images of biceps femoris long head and semitendinosus muscles were recorded by video capture EasyCap USB 2.0 (UAF Co, Limited, Shenzhen, China) in each day (intradays reliability). The gel was applied to ensure the acoustic coupling on the surface of the skin and a minimal compression of the probe under skin was adopted. The probe was positioned along the direction of the fascicles, where the fascicular organization between the superficial and deep aponeurosis on the muscle was better visualized.

In each of the images, muscle thickness, fascicle length and pennation angle variables were measured three times in random order using IMAGE J software (version 1.42; National Institute of Health, Bethesda, USA). Figure 1 explains the experimental design.

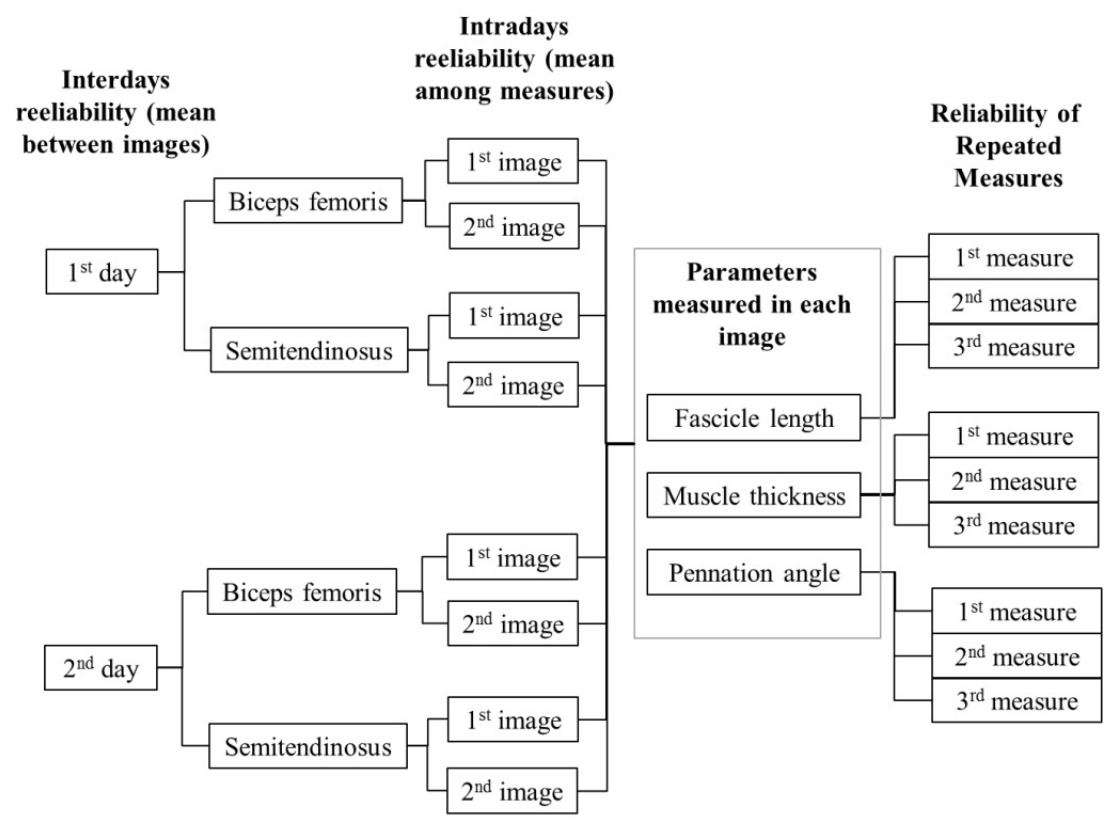

Figure 1. Experimental design for each participant. All volunteers $(\mathrm{N}=21)$ participated of two days of the test, and two images for biceps femoris and semitendinosus were obtained ( 8 images per participant). In each image, pennation angle, fascicle length and muscle thickness were repeated three times. 
Muscle thickness was measured as the mean distance between the superficial and deep aponeurosis at both image extremities. Fascicle length was estimated according to Blackburn and Pamukoff (2014). Parameters were calculated as the relative acute angle formed between the deep aponeurosis and muscle fascicle, as represented in Figure 2.

\section{Statistical analysis}

The distribution of each variable was examined with D'Agostino-Pearson test (smallest p-value found $=0.07$ ). ANOVA repeated measurements was used to test statistical differences among measurements of each parameter in the same image, to compare two images of the same days and, finally, all measurements obtained in two days. The relative reliability among three repeated measurements, intra and interdays was determined by ICC and $\alpha$ Cronbach. Model ' 2 ,k' (two-way randow factor) proposed by Shrout and Fleiss (1979) was chosen to calculate ICC. After, ICC was classified according to $\mathrm{Koo}$ and $\mathrm{Li}$ (2016) and presented with its confidence interval. $\alpha$ Cronbach was applied for estimative of internal consistency. Statistical analysis was performed using SPSS version 21 (IBM Corporation, New York, USA), with significance level of $\alpha=0.05$. The heteroskedasticity was analysed by Levene's test and Bland-Altman plots in MATLAB (version 7.10; MathWorks, USA) to present limits of agreement of data. If heteroskedasticity was present, then Bland-Altman plots were generated after logarithmic transformation of the data. Bland-Altman plots interdays were constructed to analyze graphically the distribution of data within the $95 \%$ limits of agreement and the difference between days (bias). The relative coefficient of variation (in percent - $\% \mathrm{CV}$ ) will be estimated in the presence of heteroskedasticity, otherwise relative standard error of measurement (in percent - \%SEM) will be calculated, based on the mean square error of the one-way repeated-measures ANOVA (Atkinson and Nevill, 1998; Hopkins, 2000).

\section{Results}

Among three Measurements: Averages of fascicle length, muscle thickness and pennation angle for biceps were $10.46 \pm 2.01 \mathrm{~cm} ; 2.04 \pm 0.36 \mathrm{~cm}$ and $12.52 \pm 1.92^{\circ}$, respectively. Fascicle length averages of semitendinosus were $10.15 \pm 2.18 \mathrm{~cm}$; muscle thickness $2.33 \pm 0.40 \mathrm{~cm}$ and $14.28 \pm 2.14^{\circ}$ to pennation angle. There are no significant differences among groups of the three measurements of each parameter obtained in each image acquired in two days ( $\mathrm{F}=0.003$ to 3.625 ; $\mathrm{p}=0.071$ to 0.955$)$. The $\mathrm{ICC}_{23}$ and $\alpha$ Cronbach of the three measurements are presented in Table 1. All variables presented excellent ICC for both muscles, ranging from $0.93-0.99$. The $\alpha$ Cronbach ranged from $0.95-0.99$ for fascicle length, from $0.97-0.99$ for pennation angle, and 0.99 for muscle thickness, demonstrating excellent internal consistency.

Intraday Reliability: There are no significant differences for the six measurements obtained for the same parameter in the two images in two days $(\mathrm{F}=0.017$ to $1.434 ; \mathrm{p}=0.245$ to 0.898$)$. The $\mathrm{ICC}_{2,3}$ of the images for all variables are presented in Table 2 . The ICC for all variables of biceps femoris and semitendinosus muscles were moderate to excellent (ICC from 0.70-0.95), and there is increase confidence interval. Values of $\alpha$ Cronbach also show excellent internal consistency between images.

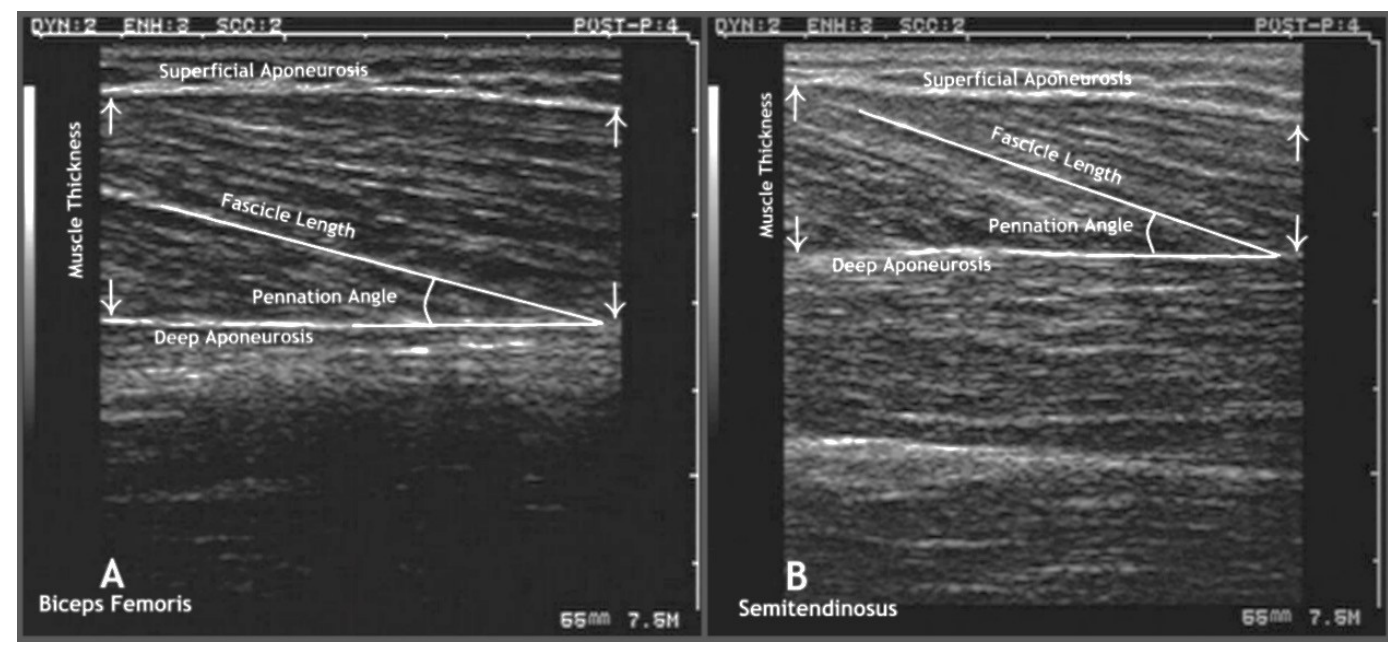

Figure 2. Ultrasound images of muscle thickness, fascicle length and pennation angle measurements of the biceps femoris long head (A) and semitendinosus (B). 
Table 1. Reliability coefficients of the three measurements.

\begin{tabular}{lllllll}
\hline & \multicolumn{2}{c}{ Fascicle length } & \multicolumn{2}{c}{ Muscle thickness } & \multicolumn{2}{c}{ Pennation angle } \\
\cline { 2 - 7 } & ICC (95\% CI) & $\boldsymbol{\alpha}$ Cronbach & ICC (95\% CI) & $\boldsymbol{\alpha}$ Cronbach & ICC (95\% CI) & $\boldsymbol{\alpha}$ Cronbach \\
\hline i1d1 & $0.95(0.88-0.98)$ & 0.95 & $0.99(0.99-0.99)$ & 0.99 & $0.96(0.91-0.98)$ & 0.98 \\
i2d1 & $0.93(0.84-0.97)$ & 0.96 & $0.99(0.99-0.99)$ & 0.99 & $0.95(0.88-0.97)$ & 0.97 \\
i1d2 & $0.94(0.87-0.97)$ & 0.97 & $0.99(0.99-0.99)$ & 0.99 & $0.95(0.89-0.98)$ & 0.97 \\
i2d2 & $0.96(0.91-0.98)$ & 0.98 & $0.99(0.99-0.99)$ & 0.99 & $0.95(0.90-0.98)$ & 0.97 \\
& & \multicolumn{7}{c}{ Bemitendinosus } & & $0.98(0.96-0.99)$ & 0.99 \\
i1d1 & $0.99(0.97-0.99)$ & 0.99 & $0.99(0.99-0.99)$ & 0.99 & 0.99 \\
i2d1 & $0.97(0.94-0.99)$ & 0.98 & $0.99(0.99-0.99)$ & 0.99 & $0.97(0.93-0.98)$ & 0.98 \\
i1d2 & $0.97(0.94-0.99)$ & 0.98 & $0.99(0.99-0.99)$ & 0.99 & $0.98(0.96-0.99)$ & 0.99 \\
i2d2 & $0.98(0.95-0.99)$ & 0.99 & $0.99(0.99-1.00)$ & 0.99 & $0.96(0.90-0.98)$ & 0.98 \\
\hline
\end{tabular}

ICC $=$ intraclass correlation coefficient; $95 \%$ CI $=95 \%$ confidence interval of the ICC; $\alpha$ Cronbach $=$ Cronbach's alpha; i1 = image $1 ;$ i2 = image $2 ; \mathrm{d} 1=$ day $1 ; \mathrm{d} 2=$ day 2 .

Table 2. Intraday reliability for fascicle length, muscle thickness and pennation angle.

\begin{tabular}{llcccccc}
\hline & \multicolumn{2}{c}{ Fascicle length } & \multicolumn{2}{c}{ Muscle thickness } & \multicolumn{2}{c}{ Pennation angle } \\
\cline { 2 - 8 } & ICC (95\% CI) & $\boldsymbol{\alpha}$ Cronbach & ICC (95\% CI) & $\boldsymbol{\alpha}$ Cronbach & ICC (95\% CI) & $\boldsymbol{\alpha}$ Cronbach \\
\hline & & & Biceps Femoris \\
Day 1 & $0.82(0.62-0.92)$ & 0.90 & $0.89(0.76-0.95)$ & 0.94 & $0.92(0.82-0.96)$ & 0.96 \\
Day 2 & $0.81(0.59-0.91)$ & 0.85 & $0.92(0.81-0.96)$ & 0.95 & $0.93(0.84-0.97)$ & 0.96 \\
& & & Semitendinosus & & & 0.93 \\
Day 1 & $0.70(0.39-0.86)$ & 0.82 & $0.95(0.89-0.98)$ & 0.97 & $0.87(0.71-0.94)$ & 0.89 \\
Day 2 & $0.81(0.60-0.92)$ & 0.89 & $0.89(0.75-0.95)$ & 0.94 & $0.81(0.59-0.91)$ & 0.94 \\
\hline
\end{tabular}

ICC $=$ intraclass correlation coefficient; $95 \% \mathrm{CI}=95 \%$ confidence interval of the ICC; $\alpha$ Cronbach $=$ Cronbach's alpha.

Interday Reliability: There are no significant differences for all the 12 measurements obtained for the same parameter in the two images along of two days ( $\mathrm{F}=0.069$ to $1.013 ; \mathrm{p}=0.326$ to 0.795 ). In all parameters there was no heteroskedasticity $(\mathrm{F}<1.03 ; \mathrm{p}>0.37)$. Moreover, SEM was determined for each parameter. Bland-Altman plots indicate good agreement for the interday measurements within $95 \%$ limits of agreement. All parameters were closer to the zero. Results of $\mathrm{ICC}_{2,3}, \alpha$ Cronbach and SEM are presented in Figure 3. The ICC was excellent for all parameters. Variables had good internal consistency $(\alpha$ Cronbach $>0.96)$.

\section{Discussion}

The present study aimed to examine the reliability of the muscle thickness, pennation angle and fascicle length measurements from ultrasonographic images from the biceps femoris long head and semitendinosus muscles. Our main results show that measurements of muscle architecture parameters presented good reliability.

The muscle architecture values are in accordance to the in vivo (Chleboun et al., 2001; Potier et al., 2009) and in vitro (Kellis et al., 2009; 2011; Ward et al., 2009) literature. Biceps femoris fascicle length values corroborate one that reported values around $10.4 \mathrm{~cm}$ in vitro (Kellis et al., 2012). On other hand, fascicle length values for semitendinosus were lower than in vitro studies (13 to $19 \mathrm{~cm}$ ) (Kellis et al., 2012; Ward et al., 2009; Woodley and Mercer, 2005). This can be explained by the large variability of muscle architecture along the whole length of the hamstring components resulting in fascicles ranging from 5.2 to $18.3 \mathrm{~cm}$ (Woodley and Mercer, 2005). Additionaly, semitendinosus is divided in two compartments separated by a tendinous inscription, and there are differences between the superficial and deep regions (Woodley and Mercer, 2005). The authors alerted that this structure has been neglected and showed that the superficial fascicles are significantly smaller than the deepest ones. We estimated the fascicle length using trigonometric extrapolation as others (Blazevich et al., 2006; Legerlotz et al., 2010), which assumes that the fascicles are linear, ignoring the curvilinear arrangements (Noorkoiv et al., 2010). This could have resulted in underestimated semitendinosus fascicle length. As far as we know, this is the first reliability in vivo study for the semitendinosus muscle.

In this work, ICC indicates repeatability and $\alpha$ Cronbach points out the internal consistency. Our results show excellent internal consistency and repeatability when considering repeated measurements 

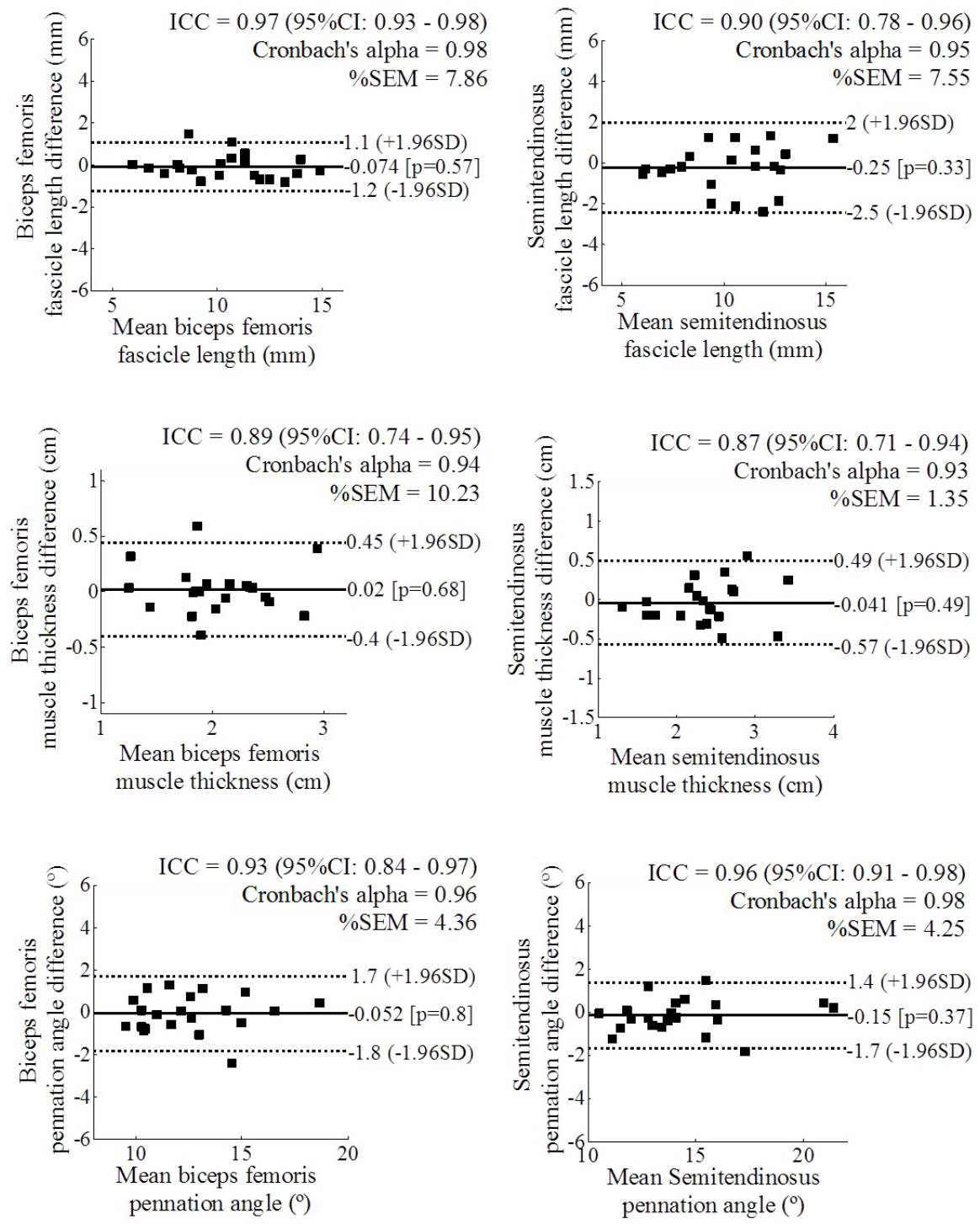

Figure 3. Bland-Altman plots of muscle architecture interdays measurements. Dashed lines represent upper and lower $95 \%$ limits of agreement. Solid lines represent the estimated bias.

in the same image for both muscles. There was a reduction of these ICC when different images at different days were analyzed followed by an increase of the confidence interval. This behavior is hardly discussed in the literature, as only the mean coefficient is reported among measurements (Gomes et al., 2010; Timmins et al., 2015). Bland-Altman plots showed small difference among measurements with bias close to zero, and almost all within limits of agreements.

Our results were similar to Timmins et al. (2015), who obtained high reproducibility $(\mathrm{ICC}>0.93$ ) and SEM of $4.9 \%$, notwithstanding they estimated the fascicle length of biceps femoris by another methodology different from ours. Chleboun et al. (2001) also demonstrated excellent reliability $(\mathrm{ICC}=0.87$ ) for biceps femoris fascicle length measurements of the in vivo. In current work, the pennation angle had excellent reliability as the data reported in others studies, ranging of 0.87 to 0.95 (Chleboun et al., 2001; Kellis et al., 2009; Timmins et al., 2015) and a SEM around 3.2 and $9.5 \%$ (Kellis et al., 2009; Timmins et al., 2015). Biceps femoris muscle thickness had excellent reliability, corroborating Timmins et al. (2015) that showed ICC of 0.95 . Gomes et al. (2010) found a 
moderate reliability $(\mathrm{ICC}=0.55, \mathrm{CV}=6.66 \%$ and $\mathrm{SEM}$ $=4.9 \mathrm{~mm}$ ) for knee flexors muscle thickness within interdays measurements. In this case, components of the flexors knee were singly evaluated.

Reliability data for semitendinosus ratifies the in vitro literature. Kellis et al. (2009) reported for fascicle length, muscle thickness and pennation angle values to ICC of $0.77,0.90$ and 0.97 , respectively, from six cadaveric limbs. The authors found SEM below $10 \%$. In vivo, high intrarater and interrater reproducibility was reported to semitendinosus pennation angle (ICC > 0.83) (Kellis et al., 2011). This study is the first to describe the in vivo muscle thickness and fascicle length measurements reliability for the semitendinosus muscle using ultrasound.

The present study indicates that muscle thickness, pennation angle and fascicle length for both biceps femoris long head and semitendinosus presented good reliability and repeatability in estimative of muscle architecture parameters by ultrasonography. The protocol of this study resulted in excellent ICC and $\alpha$ Cronbach, and these indices can to be used by future studies approaching therapeutic interventions in muscle architecture hamstring.

\section{Acknowledgements}

The authors wish to acknowledge the financial support of the Brazilian Research agencies CAPES, FINEP, FAPERJ and CNPq.

\section{References}

Atkinson G, Nevill AM. Statistical methods for assessing measurement error (reliability) in variables relevant to sports medicine. Sports Medicine. 1998; 26(4):217-38. PMid:9820922. http://dx.doi.org/10.2165/00007256199826040-00002.

Blackburn J, Pamukoff D. Geometric and architectural contributions to hamstring musculotendinous stiffness. Clinical Biomechanics. 2014; 29(1):105-10. PMid:24220042. http://dx.doi.org/10.1016/j.clinbiomech.2013.10.011.

Blazevich AJ, Gill ND, Zhou S. Intra-and intermuscular variation in human quadriceps femoris architecture assessed in vivo. Journal of Anatomy. 2006; 209(3):289310. PMid:16928199. http://dx.doi.org/10.1111/j.14697580.2006.00619.x.

Chleboun GS, France R, Crill MT, Braddock HK, Howell $\mathrm{JN}$. In vivo measurement of fascicle length and pennation angle of the human biceps femoris muscle. Cells, Tissues, Organs. 2001; 169(4):401-9. PMid:11490120. http://dx.doi. org/10.1159/000047908.

Gomes PS, Meirelles CD, Leite SP, Montenegro CA. Confiabilidade da medida de espessuras musculares pela ultrassonografia. Revista Brasileira de Medicina do
Esporte. 2010; 16(1):41-5. http://dx.doi.org/10.1590/S151786922010000100008 .

Hopkins WG. Measures of reliability in sports medicine and science. Sports Medicine. 2000; 30(1):1-15. PMid:10907753. http://dx.doi.org/10.2165/00007256-200030010-00001.

Kellis E, Galanis N, Kapetanos G, Natsis K. Architectural differences between the hamstring muscles. Journal of Electromyography and Kinesiology. 2012; 22(4):520-6. PMid:22564790. http://dx.doi.org/10.1016/j.jelekin.2012.03.012.

Kellis E, Galanis N, Natsis K, Kapetanos G. Validity of architectural properties of the hamstring muscles: correlation of ultrasound findings with cadaveric dissection. Journal of Biomechanics. 2009; 42(15):2549-54. PMid:19646698. http://dx.doi.org/10.1016/j.jbiomech.2009.07.011.

Kellis E, Galanis N, Natsis K, Kapetanos G. In vivo and in vitro examination of the tendinous inscription of the human semitendinosus muscle. Cells, Tissues, Organs. 2011; 195(4):365-76. PMid:21828998. http://dx.doi. org/10.1159/000327574.

Koo TK, Li MY. A guideline of selecting and reporting intraclass correlation coefficients for reliability research. Journal of Chiropractic Medicine. 2016; 15(2):155-63. PMid:27330520. http://dx.doi.org/10.1016/j.jcm.2016.02.012.

Legerlotz K, Smith HK, Hing WA. Variation and reliability of ultrasonographic quantification of the architecture of the medial gastrocnemius muscle in young children. Clinical Physiology and Functional Imaging. 2010; 30(3):198-205. http://dx.doi.org/10.1111/j.1475-097X.2010.00925.x. PMid:20184623.

Lieber RL. Skeletal muscle anatomy. In: Lieber RL. Skeletal muscle, structure, function, and plasticity: the physiological basis of rehabilitation. 3rd ed. Philadelphia: Lippincott Williams \& Wilkins; 2010. p. 26-41.

Lima KM, Matta TT, Oliveira LF. Reliability of the rectus femoris muscle cross-sectional area measurements by ultrasonography. Clinical Physiology and Functional Imaging. 2012; 32(3):221-6. PMid:22487157. http://dx.doi. org/10.1111/j.1475-097X.2011.01115.x.

Martins NS, Peixinho CC, Oliveira LF. Confiabilidade de medidas de arquitetura muscular do tríceps sural por ultrassonografia de imagem. Revista Brasileira de Cineantropometria \& Desempenho Humano. 2012; 14(2):212-20.

Noorkoiv M, Nosaka K, Blazevich AJ. Assessment of quadriceps muscle cross-sectional area by ultrasound extended-field-of-view imaging. European Journal of Applied Physiology. 2010; 109(4):631-9. PMid:20191287. http://dx.doi.org/10.1007/s00421-010-1402-1.

Potier T, Alexander C, Seynnes O. Effects of eccentric strength training on biceps femoris muscle architecture and knee joint range of movement. European Journal of Applied Physiology. 2009; 105(6):939-44. PMid:19271232. http:// dx.doi.org/10.1007/s00421-008-0980-7.

Shrout P, Fleiss J. Intraclass correlations: uses in assessing rater reliability. Psychological Bulletin. 1979; 86(2):420-8. PMid:18839484. http://dx.doi.org/10.1037/0033-2909.86.2.420.

Timmins RG, Shield A, Williams M, Lorenzen C, Opar D. Biceps Femoris Long-Head Architecture: a reliability and retrospective injury study. Medicine and Science in Sports 
and Exercise. 2015; 47(5):905-13. http://dx.doi.org/10.1249/ MSS.0000000000000507.

Ward SR, Eng CM, Smallwood LH, Lieber RL. Are current measurements of lower extremity muscle architecture accurate? Clinical Orthopaedics and Related Research.
2009; 467(4):1074-82. http://dx.doi.org/10.1007/s11999 008-0594-8.

Woodley S, Mercer S. Hamstring muscles: architecture and innervation. Cells Tissues Organs. 2005; 179(3):125-41. http://dx.doi.org/10.1159/000085004.

\section{Authors}

Viviane Bastos de Oliveira ${ }^{1}$, Simone Peres Carneiro ${ }^{2}$, Liliam Fernandes de Oliveira ${ }^{1,2 *}$

${ }^{1}$ Biomedical Engineering Program, Instituto Alberto Luiz Coimbra de Pós-Graduação e Pesquisa em Engenharia COPPE, Centro de Tecnologia, Universidade Federal do Rio de Janeiro - UFRJ, Avenida Horácio Macedo, 2030, Cidade Universitária, CEP 21941-598, Rio de Janeiro, RJ, Brazil.

${ }^{2}$ School of Physical Education and Sports, Department of Biosciences, Universidade Federal do Rio de Janeiro - UFRJ, Rio de Janeiro, RJ, Brazil. 